\title{
AstraZeneca Pharmaceuticals responds to the chronic obstructive pulmonary disease crisis with the launch of a non-interventional study - COPVAR
}

According to World Health Organization (WHO) reports, chronic obstructive pulmonary disease (COPD) is the third-leading cause of deaths worldwide. ${ }^{[1]}$ Further, it is the sixth-leading cause of disabilityadjusted life years (DALYs) in middle-income countries - a figure that is expected to change to the fifth-leading cause worldwide in 2030 - and the significance of the disease is becoming quite evident. In response to this, AstraZeneca, a global, research-based biopharmaceutical company with a vested interest in the development of life-changing respiratory medications, has launched a cross-sectional study of patients with severe COPD that assesses patient perception of symptom variability in Middle East and African (MEA) countries.

COPD is characterised by persistent airflow limitation associated with a chronic inflammation of the small airways. ${ }^{[2]}$ This is manifested in multiple respiratory symptoms, including dyspnoea, cough, sputum production, wheezing and chest tightness. Anorexia, fatigue and weight loss may also be expressed in more severe disease. AstraZeneca's non-interventional cross-sectional study of Global Initiative for Chronic Obstructive Lung Disease (GOLD) risk category $\mathrm{C}$ and $\mathrm{D}$ patients with severe COPD is primarily intended to assess patient perception of daily and weekly symptoms and their impact on daily life activities.

According to Dr Jasvanti Bhana, AstraZeneca South Africa (SA) Vice President for Medical and Regulatory Affairs, it is believed that the spike in COPD mortality rates is directly linked to the expanding smoking epidemic, exposure to biomass fuel (particularly in the developing world), reduced mortality from other common causes of death (ischaemic heart disease, infectious diseases) and the aging of the world population. Adding to this, she states that regardless of the cause and contributing factors, AstraZeneca is a research-based organisation and committed to being part of a solution that eases the healthcare burden through studies such as COPVAR. 'Although COPD symptoms have been extensively reviewed in the literature, daily and weekly variation of symptoms and their impact on daily life activities have received less attention, especially in the MEA region,' says Bhana. 'No published study in MEA has investigated the fluctuation of COPD symptoms, the COPD treatment patterns, and their consistency with the GOLD guidelines.'

This non-interventional study also aims to explore current practice in the management of stable GOLD C and D COPD patients. 'On conclusion of this study we must be able to describe the current treatment practice for the management of GOLD C and D COPD in MEA countries and their adherence to the GOLD 2016 guidelines,' says Bhana. 'We also want to draw comparisons between GOLD categories $\mathrm{C}$ and $\mathrm{D}$ to uncover patient perceptions of symptom variability and their impact. The identification of demographic, behavioural and clinical factors associated with symptom variability is another important study outcome sought by our [non-interventional study] NIS researchers.'
With SA now included in the study, there are nine participating countries. The other countries taking part are Algeria, Egypt, Jordan, Kuwait, Lebanon, Qatar, Turkey and the United Arab Emirates. It is expected that approximately 3085 stable GOLD C and D COPD patients will be recruited during the 12-month multicentre study, of whom 350 will be SA patients. 'Data collation - directly gathered from patients via self-administered questionnaires - will take place during a one-visit assessment; no follow-ups will be performed. By design, there also are no specific tests or examinations carried out for the purpose of the study.' Bhana concludes, '[Research and development] R\&D is the life blood of medical solutions that save lives. Despite its pressures, we embrace the significant scientific leadership role we have achieved as a result of ethical, high quality research outputs, and SA is at the forefront of research for our Group.'

For 40 years, AstraZeneca has pushed the boundaries of science in respiratory disease. Starting with our first bronchodilator, through to our currently marketed products and a pipeline of respiratory products for both asthma and COPD, AstraZeneca scientists continue to transform disease management and patient outcomes in asthma and COPD. AstraZeneca continues to follow the science by developing targeted inhaled and biological therapies, unique drug combinations, and devices to ensure the right treatments for the right patients.

For further information on the COPVAR study contact AstraZeneca: AstraZeneca Pharmaceuticals (Pty) Ltd. South Africa Reg. No. 1992/005854/07

Building 2, Northdowns Office Park, 17 Georgian Crescent West, Bryanston, 2191, South Africa. Private Bag X23, Bryanston, 2021, South Africa.

Tel: +27 (0)11 797 6000. Fax: +27 (0)11 7976001 .

www.astrazeneca.com.

1. World Health Organization. Fact sheets. Geneva: WHO, 2014. http://www.who.int/ mediacentre/factsheets/fs310/en/ (accessed November 2014).

2. Global Initiative for Chronic Obstructive Lung Disease. Global strategy for the diagnosis, management and prevention of chronic obstructive pulmonary disease. 2014. http://goldcopd.org/ (accessed November 2014).

\section{Notes}

Disease area

COPD patients may experience acute intensifications of symptoms requiring additional treatment, commonly called exacerbations (Burge \& Wedzicha, 2003).

Grading and exacerbations

Although COPD is considered a stable condition in the absence of exacerbations, there is recent evidence of weekly and daily variability in symptom perception (Kessler et al., 2011). 
GOLD recommends grading disease severity into risk groups (A - D) based on symptoms, lung function and exacerbation risk. Symptoms are assessed by COPD assessment test (CAT) or modified Medical Research Council (mMRC) dyspnoea scale. A CAT $\geq 10$ indicates high impact of symptoms (risk groups B and D). Exacerbation risk is determined by the degree of airflow limitation using lung function impairment grades I - IV or by the number of exacerbations in the previous 12 months. Patients with a forced expiratory volume in 1 second $<50 \%$ predicted and those with $\geq 2$ past-year exacerbations or $>1$ exacerbation that leads to hospitalisation have a high exacerbation risk (risk group C and D).

Adding to exacerbations, patients have reported experiencing seasonal, weekly or even daily worsening of symptoms not consistently fitting an exacerbation episode. The foregoing perceived symptom variability is a factor directly affecting disease impact on daily activities, quality of life and prognosis (Lopez-Campos, Calero \& Quintana-Gallego, 2013).
COPD symptom variability and its effect on patients have been substantially examined. The first study to document daily variability in symptoms was an internet interview of 803 COPD patients in Europe and USA. Forty-six percent of the interviewed patients reported that morning was the worst time of the day, and shortness of breath was the most reported symptom, which was highly correlated with the reported limitations encountered during morning periods (Partridge, Karlsson \& Small, 2009).

In addition, a pan-European cross-sectional study that aimed to observe COPD symptom variability in daily life surveyed 2441 patients from 17 countries during a 7-day period. Of the patients, $72.5 \%$ reported experiencing dyspnoea, among other symptoms, and $59.5 \%$ perceived seasonal variability with symptoms worsening (NIS Primary Protocol (NISP) NIS D-code: D2287R00105, edition number: V1.0, date: 16 July 2015, 11(40)). 